\title{
Efficacy and safety of ifosfamide-based chemotherapy for osteosarcoma: a meta-analysis
}

This article was published in the following Dove Press journal:

Drug Design, Development and Therapy

4 November 2015

Number of times this article has been viewed

\section{Xiao-Liang Fan ${ }^{1, *}$ \\ Guo-Ping Cai ${ }^{2}, *$ \\ Liu-Long Zhu' \\ Guo-Ming Ding'}

'Department of Orthopaedics, Hangzhou First People's Hospital, Nanjing Medical University, Hangzhou, ${ }^{2}$ Department of Orthopaedics, Jinshan Hospital, Fudan University, Shanghai,

People's Republic of China

*These authors contributed equally to this work
Correspondence: Xiao-Liang Fan Department of Orthopaedics, Hangzhou First People's Hospital, Nanjing Medical University, 26I Huansha Road, Hangzhou 310006 , People's Republic of China Tel 8605 I9 |880 57/5 I9| Email fanxliang@।26.com
Background: The efficacy of ifosfamide-based chemotherapy in the treatment of osteosarcoma has been investigated; however, results are inconsistent. Therefore, we reviewed the relevant studies and conducted a meta-analysis to assess the efficacy of ifosfamide-based chemotherapy in patients with osteosarcoma.

Methods: A systematic literature search on PubMed, Embase, and Web of Science databases was performed. Eligible studies were clinical trials of patients with osteosarcoma who received ifosfamide-based chemotherapy. Hazard ratios (HRs) were pooled to compare event-free survival (EFS) and overall survival (OS). Risk ratios (RRs) were pooled to compare good histologic response rates and adverse event incidence. Meta-analysis was performed using a fixed-effects model or a random-effects model according to heterogeneity.

Results: A total of seven randomized controlled trials were included in this meta-analysis. Pooled results showed that ifosfamide-based chemotherapy significantly improved EFS (HR=0.72, 95\% confidence interval $[\mathrm{CI}]: 0.63,0.82 ; P=0.000)$ and $\mathrm{OS}(\mathrm{HR}=0.83,95 \% \mathrm{CI}: 0.70,0.99$; $P=0.034)$; furthermore, this form of chemotherapy increased good histologic response rate ( $\mathrm{RR}=1.27,95 \% \mathrm{CI}: 1.10,1.46 ; P=0.001)$. In addition, patients in the ifosfamide group exhibited a significantly higher incidence of fever $(\mathrm{RR}=2.23,95 \% \mathrm{CI}: 1.42,3.50 ; P=0.000)$ and required more frequent platelet transfusion $(\mathrm{RR}=1.92,95 \% \mathrm{CI}: 1.23,3.01 ; P=0.004)$.

Conclusion: This meta-analysis confirmed that ifosfamide-based chemotherapy can significantly improve EFS and OS; this chemotherapy can also increase good histologic response rate in patients with osteosarcoma. However, evidence may be limited by potential biases and confounders. Thus, large-scale well-designed randomized controlled trials are needed to verify current findings.

Keywords: ifosfamide, osteosarcoma, chemotherapy, meta-analysis

\section{Introduction}

Osteosarcoma is a pleomorphic malignant tumor of the bone that occurs in children, adolescents, and young adults. ${ }^{1}$ This condition can develop in any bone but most commonly occur in the metaphyses of long bones. Chemotherapy and surgery can result in $\sim 60 \%-70 \% 5$-year survival of patients who do not suffer from metastases. In contrast, the overall survival (OS) of patients who present metastatic disease decreases to $\sim 30 \% .^{2}$ Despite several advanced approaches, including surgery, radiotherapy, and chemotherapy, which have been applied to improve the outcomes of patients with osteosarcoma, long-term survival has slightly improved in the past decades.

In the early 1980s, ifosfamide, with or without etoposide, has been used in combination with chemotherapy to enhance the survival of patients with osteosarcoma. ${ }^{3-7}$ Patients who experienced relapse after standard therapies for osteosarcoma have shown remarkable responses when they were subjected to ifosfamide-based chemotherapy. ${ }^{3-7}$ In a Phase II study of the French Society of Pediatric Oncology involving patients with 
relapsed or refractory osteosarcoma, ${ }^{8}$ the combined therapy of ifosfamide and etoposide resulted in a $48 \%$ response rate (95\% confidence interval (CI): 29\%, 67\%). However, these results are in contrast to those from the studies of two multiinstitutional groups, namely, the German/Dutch/Austrian/ Swiss Cooperative Ewing's Sarcoma Studies (from the Gesellschaft fur PadiatrischeOnkologie und Hamatologie) and UK Children's Cancer Study Group. ${ }^{9,10}$ In these two studies, patients were assigned to receive neoadjuvant with or without ifosfamide. However, no significant difference in survival was found. Therefore, a meta-analysis of all available studies was conducted to assess the efficacy and safety of ifosfamide-based chemotherapy for patients with osteosarcoma.

\section{Methods}

\section{Literature search strategy}

This meta-analysis was performed according to the Preferred Reporting Items for Systematic Reviews and Meta-Analysis criteria. ${ }^{11}$ PubMed, Embase, and Web of Science databases were searched for original articles published before April 4, 2015. The literature search was updated on July 27, 2015. The search was limited to human subjects; no language restriction was imposed. Abstracts presented at the annual meeting of the American Society of Clinical Oncology and the European Cancer Conference were also searched. Moreover, we manually searched the reference lists of eligible studies until no potential studies could be further found. The following initial search items were used: "osteosarcoma” [MeSH Terms] OR "osteosarcoma" [All Fields] AND "drug therapy" [Subheading] OR "drug" [All Fields] AND "therapy" [All Fields] OR "drug therapy" [All Fields] OR "chemotherapy" [All Fields] OR "drug therapy" [MeSH Terms] OR "drug” [All Fields] AND "therapy" [All Fields] OR "chemotherapy" [All Fields] AND "ifosfamide" [MeSH Terms] OR "ifosfamide" [All Fields].

\section{Review strategy and inclusion criteria}

Endnote (version X, Thomson Reuters, Inc., Philadelphia, PA, USA) bibliographic software was used to create an electronic library of citations identified in the database searches. The literature searches were conducted using Endnote, and duplicate records were deleted. Two investigators (X-LF and G-PC) independently performed the title/abstract review, and then the full-text review. Disagreements between the two investigators were resolved by consensus and discussion.

Studies that met the following inclusion criteria were considered eligible for this meta-analysis: 1) study subjects, patients with histologically confirmed osteosarcoma; 2) study intervention, patients in the experimental group received ifosfamide-based chemotherapy, whereas patients in the control group received chemotherapy without ifosfamide; 3) outcome measures, included event-free survival (EFS), OS, good histologic response rate, and toxicity. Reviews, comments, case report, editorials, letters, or articles unrelated with our topics were excluded from final analysis.

\section{Data extraction and quality assessment}

Two independent investigators (X-LF and G-PC) extracted the following information from the selected studies: first author, year of publication, country, sample size in each group, regimens, EFS, OS, and toxicity. In cases where the same trial appeared in different publications, studies with the most relevant information or the longest follow-up period were included.

The quality of randomized controlled trials (RCTs) was evaluated using the Jadad scale. ${ }^{12}$ The scale consists of three items, including randomization ( $0-2$ points), blinding ( $0-2$ points), and dropouts and withdraws ( $0-1$ point), to report the quality of a RCT. A score of 1 is obtained for each of the items described. A further point is given when the method of randomization or blinding is described. The total score of Jadad scale is 5 points. Studies with a total score $\geq 3$ points are considered of high quality. ${ }^{13}$

\section{Statistical analysis}

This meta-analysis was conducted using STATA version 12.0 (StataCorp LP, College Station, TX, USA). A fixedeffects model ${ }^{14}$ or random-effects model ${ }^{15}$ was used to pool the estimates when heterogeneity among studies was absent or present. Heterogeneity was tested using Cochran's Q chi-square test and $I^{2}$ statistic. A $P$-value $<0.1$ or $I^{2}>50 \%{ }^{16}$ were defined to have heterogeneity. The EFS and OS were treated as time-to-event variables; thus, these parameters were expressed as hazard ratios (HRs) with 95\% CI for each study. In some studies, Kaplan-Meier curves instead of HRs with $95 \%$ CI were provided; thus, HRs with $95 \%$ CI were calculated from Kaplan-Meier curves using the method described by Tierney. ${ }^{17}$ Good histologic response rate and toxic event incidence were considered dichotomous variables; thus, these parameters were expressed as risk ratios (RRs) with 95\% CI for each study. Publication bias was evaluated using the Begg's and Egger's test. ${ }^{18,19}$ A two-tailed $P$-value $<0.05$ was considered statistically significant.

\section{Results}

\section{Identification of eligible studies}

A total of 361 potential articles were identified from the databases search. Among these articles, 87 were excluded after duplicate review, and 217 were excluded after title/abstract 
review, leaving 57 articles for the full-text review (Figure 1). In the review, 50 studies were excluded for the reasons as follows: three were eliminated because their data were unavailable for analysis, seven studies were of single-arm designs, three studies involved blood stem cell rescue in the intervention group, and one study presented overlapping data with another study. Finally, seven studies with a total of 2,529 patients that met the inclusion criteria were included in this meta-analysis. ${ }^{20-26}$

\section{Study characteristics and quality assessment}

The baseline characteristics of the included studies are presented in Table 1. Among the seven studies conducted between 2003 and 2012, all were RCTs. The patients in these studies were from Japan, Italy, France, UK, and US. Primary tumors were located in the femur $(46.5 \%)$ and in the tibia $(21.8 \%)$ of the patients. Approximately $1.9 \%$ of the patients suffered from metastatic osteosarcoma when they were enrolled in the studies. The median follow-up among these studies ranged from 66 to 102 months. Treatment regimen among the studies varied greatly; however, chemotherapy in most of the experimental groups consisted of etoposide, methotrexate (MTX), cisplatin, and doxorubicin. The dose of ifosfamide administrated for patients ranged from 2 to $9 \mathrm{~g} / \mathrm{m}^{2} /$ day, and in most of the studies, the ifosfamide was administrated for 3 days. The median Jadad scale of the RCTs was 3 (range: from 3 to 4 ).

\section{Event-free survival}

Seven studies provided the data of EFS. ${ }^{20,21-26}$ Meta-analysis of all the studies using a fixed-effects model showed that ifosfamide-based chemotherapy significantly improved the EFS $(\mathrm{HR}=0.72,95 \% \mathrm{CI}: 0.63,0.82 ; P=0.000)$ in the treatment of osteosarcoma (Figure 2), with no statistically significant heterogeneity between individual trials $\left(P=0.357, I^{2}=9.2 \%\right)$. Considering that this meta-analysis was performed on the basis of different sample sizes, we therefore performed sensitivity analyses to identify whether this result was influenced by these factors. Similar results were obtained when the trial with modest sample sizes $(\mathrm{N} \leq 100)$ was excluded $(\mathrm{HR}=0.71$, 95\% CI: 0.61, 0.81, $P=0.000$; heterogeneity test: $P=0.299$, $\left.I^{2}=18.2 \%\right) .{ }^{23}$ Begg's and Egger's test indicated the absence of publication bias (for Begg's test: $Z=0.00, P=1.000$; for Egger's test: $t=0.03, P=0.975$ ).

We also attempted to perform subgroup analysis to explore whether the EFS results of ifosfamide would differ in patients with different tumor sites, tumor sizes, and

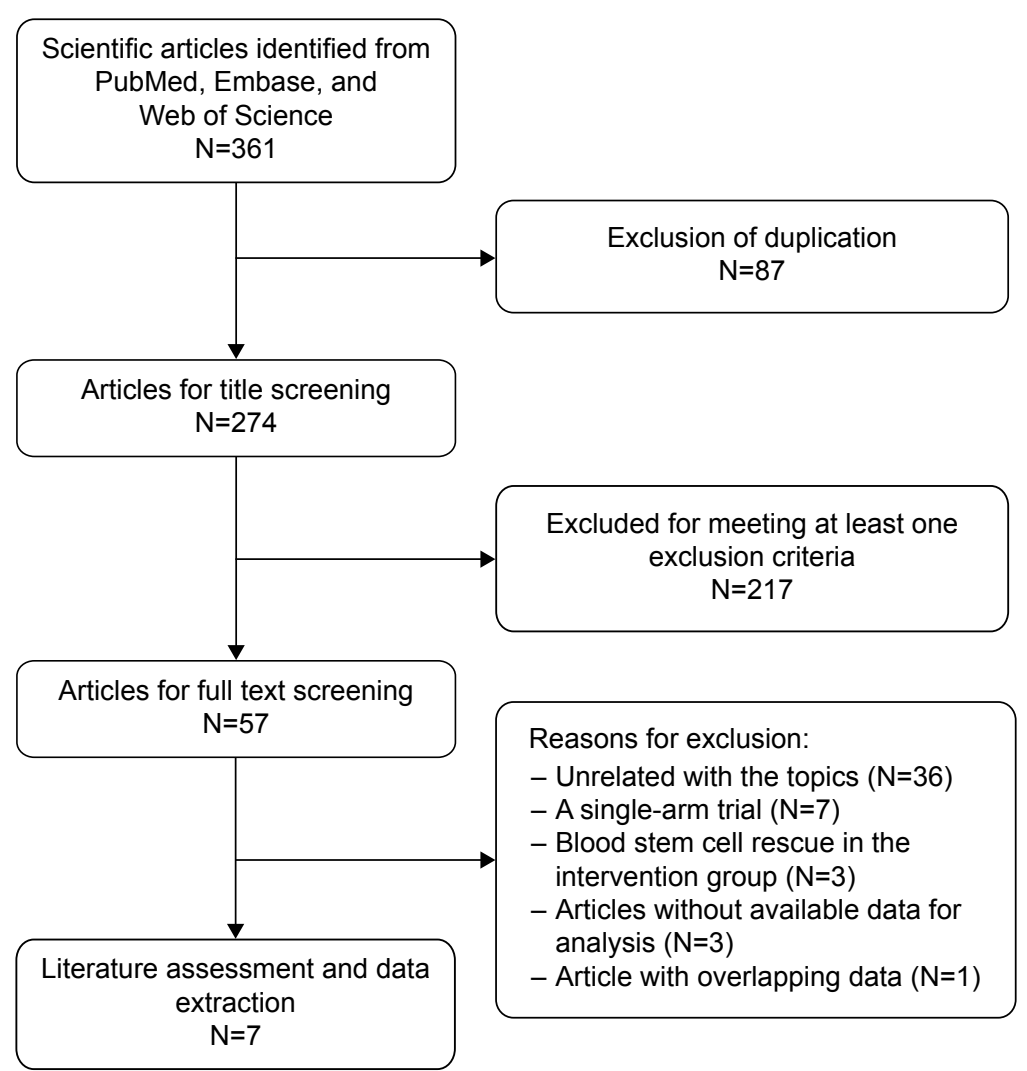

Figure I Search strategy and flow chart for this meta-analysis. 
Table I Baseline characteristics of patients in the trials included in the meta-analysis

\begin{tabular}{|c|c|c|c|c|c|c|c|c|}
\hline Author & $\begin{array}{l}\text { Study } \\
\text { design }\end{array}$ & Treatment regimen & $\begin{array}{l}\text { No of } \\
\text { patients }\end{array}$ & $\begin{array}{l}\text { Median age } \\
\text { (year, range) }\end{array}$ & $\begin{array}{l}\text { Male/ } \\
\text { female }\end{array}$ & $\begin{array}{l}\text { Tumor location } \\
\text { (femur/tibia/ } \\
\text { humerus/other) }\end{array}$ & $\begin{array}{l}\text { Metastases } \\
\text { (yes/no) }\end{array}$ & $\begin{array}{l}\text { Jadad } \\
\text { scale }\end{array}$ \\
\hline \multirow[t]{2}{*}{ Le Deley et $\mathrm{al}^{20}$} & $\mathrm{RCT}$ & MTX + ETO + IFO & 118 & $13.3(5.5-19.3)$ & $72 / 46$ & $55 / 27 / 11 / 25$ & $0 / 118$ & 3 \\
\hline & & MTX + DOX & 116 & $13.2(3.1-19.5)$ & $59 / 57$ & $52 / 23 / 16 / 24$ & $0 / 116$ & \\
\hline \multirow[t]{2}{*}{ Meyers et $\mathrm{a}^{21}$} & $\mathrm{RCT}$ & $\mathrm{DOX}+\mathrm{CDP}+\mathrm{HDMTX}$ & 677 & $13(1-30)$ & $373 / 304^{*}$ & $374 / 185 / 73 / 45^{*}$ & $0 / 377$ & 3 \\
\hline & & DOX + IFO + HDMTX & & $13(I-30)$ & & & & \\
\hline \multirow[t]{2}{*}{ Bacci et $\mathrm{a}^{22}$} & RCT & HDMTX & 142 & & $88 / 54$ & $80 / N R / N R / 62$ & $23 / 119$ & 3 \\
\hline & & HDMTX + IFO & 79 & & $42 / 37$ & $50 / N R / N R / 0$ & $6 / 73$ & \\
\hline \multirow[t]{2}{*}{ Chou et $\mathrm{a}^{23}$} & $\mathrm{RCT}$ & CDP, DOX, MTX, IFO & 46 & $1-30$ & $28 / 18$ & $29 / 16 / 7 / 39 *$ & $20 / 62^{*}$ & 3 \\
\hline & & CDP, DOX, MTX & 45 & $1-30$ & $28 / 17$ & & & \\
\hline \multirow[t]{2}{*}{ Meyers et $\mathrm{a}^{24}$} & $\mathrm{RCT}$ & CDP, DOX, MTX, IFO & $662 *$ & $13(1-30)$ & $361 / 301 *$ & $364 / 184 / 72 / 42^{*}$ & $\mathrm{NR}^{*}$ & 3 \\
\hline & & CDP, DOX, MTX & & $13(1-30)$ & & & & \\
\hline \multirow[t]{2}{*}{ Grier et $\mathrm{a}^{25}$} & $\mathrm{RCT}$ & VCR, CYC, DOX, IFO & 198 & NR & $120 / 78$ & $35 / 19 / 16 / 127$ & $0 / 198$ & 3 \\
\hline & & VCR, CYC, DOX & 200 & NR & $106 / 94$ & $38 / 19 / 13 / 130$ & $0 / 200$ & \\
\hline \multirow[t]{2}{*}{ Ferrari et $\mathrm{al}^{26}$} & $\mathrm{RCT}$ & MTX, CDP, DOX, IFO & 123 & 14 (6-39) & $74 / 49$ & $62 / 34 / 14 / 13$ & $0 / 123$ & 4 \\
\hline & & MTX, CDP, DOX & 123 & $14(4-34)$ & $72 / 51$ & $69 / 26 / 20 / 8$ & $0 / 123$ & \\
\hline
\end{tabular}

Note: *Data for all the patients.

Abbreviations: CDP, cisplatin; CYC, cyclophosphamide; DOX, doxorubicin; ETO, etoposide; HDMTX, high-dose methotrexate; IFO, ifosfamide; MTX, methotrexate; NR, not reported; RCT, randomized controlled trial; VCR, vincristine.

metastatic. However, no sufficient data were provided by these eligible studies for subgroup analysis. Thus, subgroup analysis was not performed.

\section{Overall survival}

Among the seven trials included in the meta-analysis, five provided data of OS..$^{20,21,23,24,26}$ The pooled analysis using a fixed-effects model suggested that ifosfamide-based chemotherapy significantly improved the OS (HR $=0.83,95 \%$ CI: $0.70,0.99 ; P=0.034)$ in the treatment of osteosarcoma (Figure 3), with no statistically significant heterogeneity between individual trials $\left(P=0.437, I^{2}=0.0 \%\right)$. We also performed sensitivity analysis. Exclusion of the study with modest sample sizes (HR=0.81, 95\% CI: 0.67, 0.97, $P=0.020$; heterogeneity test: $P=0.423, P=0.0 \%$ ), changed the overall estimate slightly, in which a marginally significant difference was observed.

Begg's and Egger's tests indicated no existence of publication bias (for Begg's test: $Z=1.22, P=0.221$; for Egger's test: $t=4.28, P=0.23)$.

\section{Good response rate}

Three studies reported the data of good histologic response rates. ${ }^{20,21,26}$ Pooled the results using a fixed-effects model

\begin{tabular}{|c|c|c|}
\hline Study ID & HR $(95 \% \mathrm{Cl})$ & $\%$ weight \\
\hline Le Deley et $a^{20}$ & $0.71(0.50,1.06)$ & 13.19 \\
\hline Meyers et $\mathrm{al}^{21}$ & $0.56(0.40,0.79)$ & 16.07 \\
\hline Chou et $\mathrm{al}^{23}$ & $0.85(0.55,1.33)$ & 9.55 \\
\hline Meyers et $\mathrm{al}^{24}$ & $0.80(0.62,1.00)$ & 32.58 \\
\hline Grier et $\mathrm{al}^{25}$ & $0.60(0.44,0.84)$ & 17.81 \\
\hline Ferrari et $\mathrm{al}^{26}$ & $0.88(0.58,1.33)$ & 10.81 \\
\hline Overall $\left(I^{2}=9.2 \%, P=0.357\right)$ & $0.72(0.63,0.82)$ & 100 \\
\hline
\end{tabular}

Figure 2 Comparison of regimens with or without ifosfamide for osteosarcoma patients in terms of EFS. Abbreviations: $\mathrm{Cl}$, confidence interval; EFS, event-free survival; $\mathrm{HR}$, hazard ratio. 


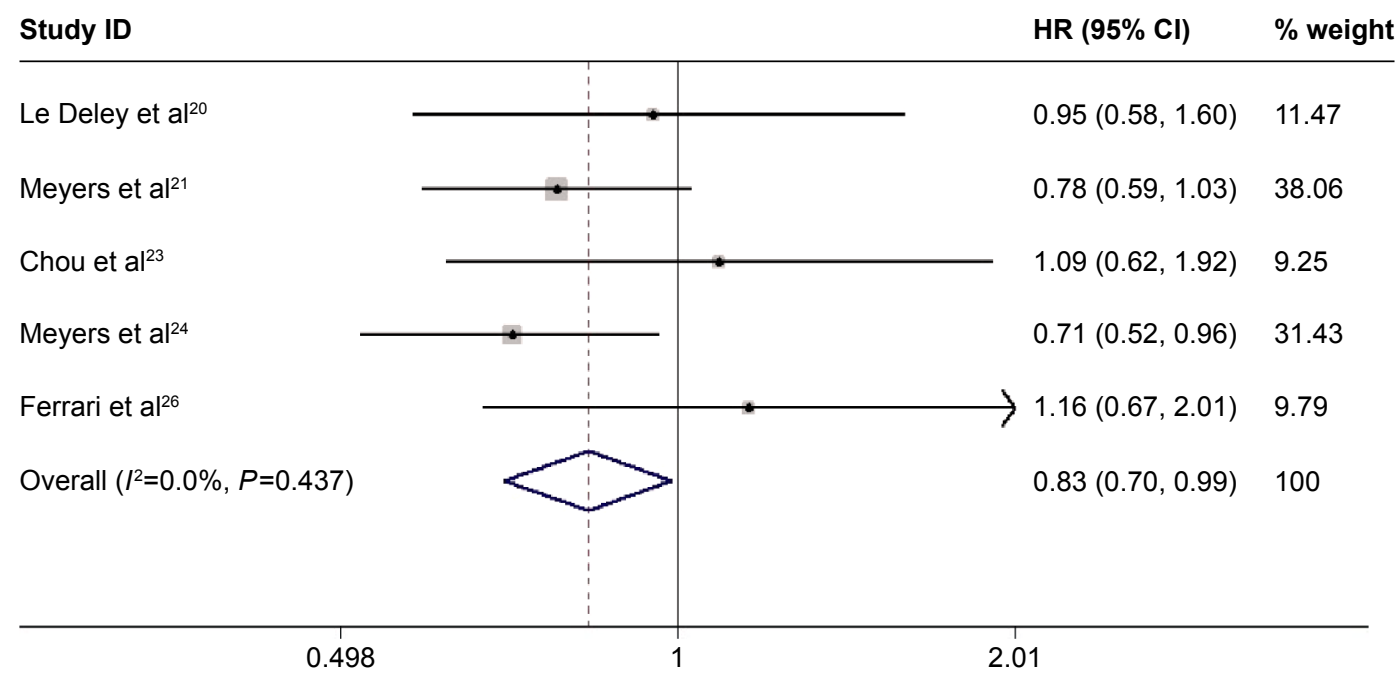

Figure 3 Comparison of regimens with or without ifosfamide for osteosarcoma patients in terms of overall survival (OS).

Abbreviations: $\mathrm{Cl}$, confidence interval; $\mathrm{HR}$, hazard ratio.

indicated that patients who received ifosfamide-based chemotherapy exhibited a higher good histologic response rate $(\mathrm{RR}=1.27,95 \% \mathrm{CI}: 1.10,1.46 ; P=0.001)$ (Figure 4) than those treated without ifosfamide. Publication bias was not assessed because the number of included studies was $<5$.

\section{Prognostic factors}

Three studies presented the data of prognostic factors. ${ }^{22,23,25}$ The pooled analysis using a random-effects model showed that these important variables known at the study center, including sex, age, tumor size, and tumor site, may not influence the EFS of patients with osteosarcoma. Female patients did not experience longer EFS than male patients $(\mathrm{RR}=1.05,95 \% \mathrm{CI}$ : $0.72,1.54 ; P=0.796)$. Moreover, children older than 16 years did not fare better than adults ( $R R=0.90,95 \%$ CI: 0.65, 1.24; $P=0.518$ ). Likewise, patients with $<10 \mathrm{~cm}$ tumor $(\mathrm{RR}=0.93$, $95 \%$ CI: $0.60,1.44 ; P=0.738$ ), or with osteosarcomas of the extremities ( $\mathrm{RR}=1.14,95 \% \mathrm{CI}: 0.98,1.33 ; P=0.082)$ did not experience prolonged EFS (Figure 5).

\section{Adverse reactions}

Four studies reported the data of adverse events, ${ }^{20,23,25,26}$ including fever, vomiting, cardiotoxicity, nervous-centcerebellar, skin toxicity, infection, leukocytopenia, and thrombocytopenia. Pooled results showed that patients subjected to ifosfamide-based chemotherapy exhibited significantly higher fever incidence $(\mathrm{RR}=2.23,95 \% \mathrm{CI}: 1.42$, $3.50 ; P=0.000)$, and required more frequent platelet transfusion $(\mathrm{RR}=1.92,95 \% \mathrm{CI}: 1.23,3.01 ; P=0.004)$.

\begin{tabular}{|c|c|c|c|}
\hline Study ID & & RR (95\% Cl) & $\%$ weight \\
\hline Le Deley et $\mathrm{al}^{20}$ & $\rightarrow$ & $1.44(1.09,1.91)$ & 28.48 \\
\hline Bacci et $\mathrm{al}^{22}$ & & $1.23(1.01,1.49)$ & 39.52 \\
\hline Ferrari et $\mathrm{al}^{26}$ & & $1.16(0.88,1.53)$ & 32.00 \\
\hline Overall $\left(I^{2}=0.0 \%, P=0.513\right)$ & & $1.27(1.10,1.46)$ & 100 \\
\hline
\end{tabular}

Figure 4 Comparison of regimens with or without ifosfamide for osteosarcoma patients in terms of good histologic response rate. Abbreviations: $\mathrm{Cl}$, confidence interval; $\mathrm{RR}$, risk ratio. 


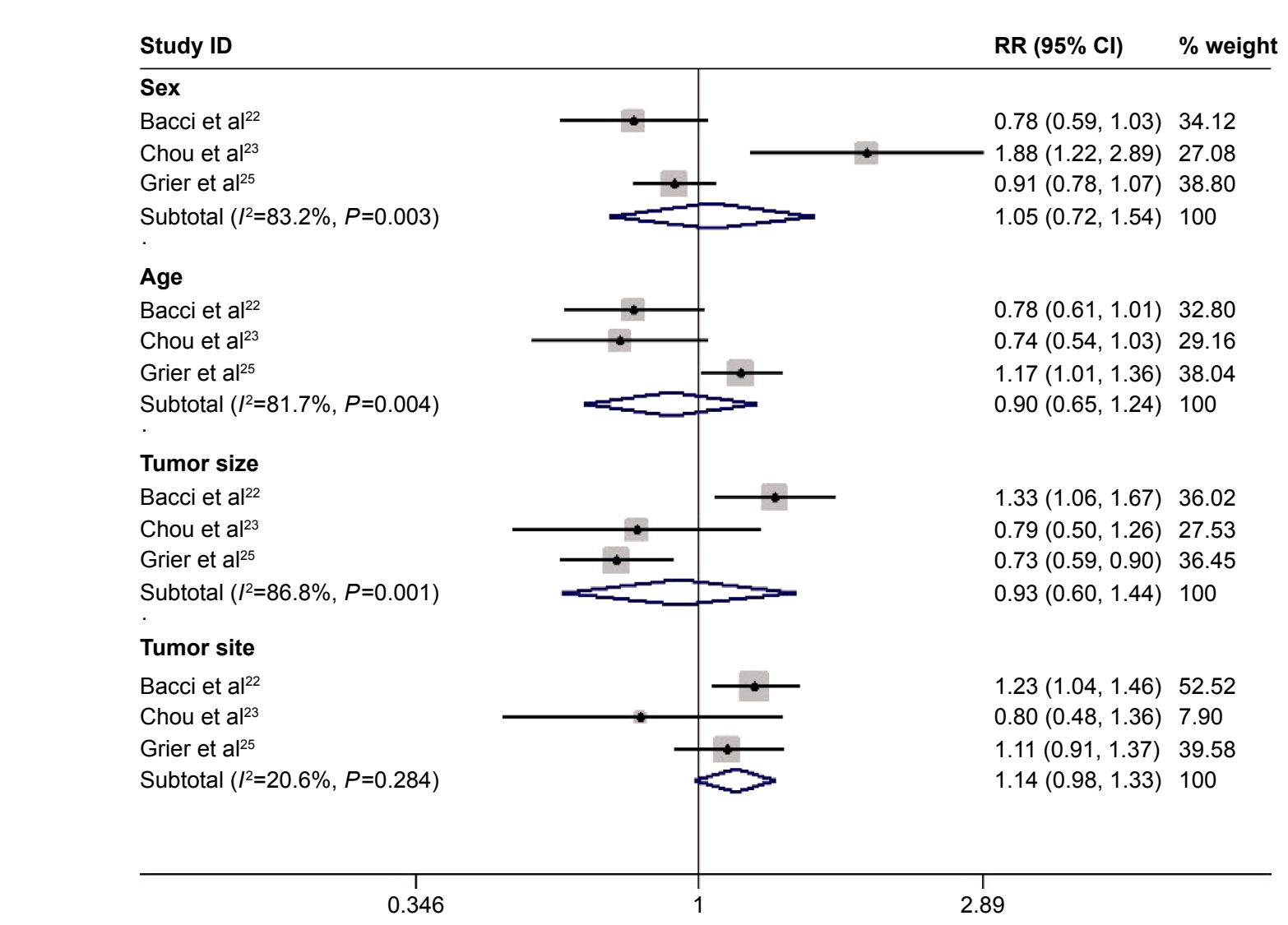

Figure 5 Comparison of regimens with or without ifosfamide for osteosarcoma patients in terms of prognostic factors.

Note: Weights are from random-effects analysis.

Abbreviations: $\mathrm{Cl}$, confidence interval; $\mathrm{RR}$, risk ratio.

\section{Discussion}

This study is a meta-analysis with the objective of assessing the efficacy and safety of ifosfamide-based chemotherapy for patients with osteosarcoma. Based on seven RCTs, the results from our meta-analysis suggested that the addition of ifosfamide to chemotherapy could significantly improve the EFS (HR $=0.72,95 \%$ CI: $0.63,0.82 ; P=0.000)$ and the OS (HR $=0.83,95 \%$ CI: $0.70,0.99 ; P=0.034$ ) of patients with osteosarcoma. Moreover, patients who received ifosfamidebased chemotherapy exhibited higher good histologic response rate $(\mathrm{RR}=1.27,95 \% \mathrm{CI}: 1.10,1.46 ; P=0.001)$ than those treated without ifosfamide. These results confirmed the significant survival benefits of ifosfamide-based chemotherapy for osteosarcoma.

In this meta-analysis, ifosfamide-based chemotherapy reduced the risk of death in patients with osteosarcoma by $17 \%$ (HR $=0.83,95 \%$ CI: $0.70,0.99 ; P=0.034)$. However, this survival benefit was only observed in the RCT conducted by Meyers et al. ${ }^{24}$ In this randomized, prospective trial, the patients were assigned to receive three-drug chemotherapy with cisplatin, doxorubicin, and MTX, or four-drug chemotherapy with cisplatin, doxorubicin, MTX, and ifosfamide. ${ }^{24}$
The authors found that 6-year OS rate in experimental and standard groups were $78 \%$ and $70 \%$, respectively. To identify whether this survival benefit was influenced by some potential factors, including number and sites of relapse and extent of surgical resection of metastases, the authors conducted a marginal analysis within a $2 \times 2$ factorial design. However, significant differences in these factors were not observed between the groups. Thus, the authors postulated that the significant improvement in OS could be attributed to the addition of ifosfamide to standard chemotherapy.

In the two trials ${ }^{25,26}$ included in this study, the effect of ifosfamide-based chemotherapy was greater in patients who did not suffer from metastatic disease than those who experienced metastases. Interestingly, both of these trials contained ifosfamide and etoposide in experimental group. In the EICESS-92 study conducted by Paulussen et al, ${ }^{27}$ the corresponding HRs for OS among the patients without and with metastases were $0.80(95 \% \mathrm{CI}=0.57,1.13 ; P=0.21)$ and $1.02(95 \% \mathrm{CI}=0.69,1.50 ; P=0.91)$, respectively. In the INT-0091 trial conducted by Grier et al, ${ }^{25}$ the relative risk of death of the patients without and with metastases who were subjected to the standard regimen were $1.6(95 \% \mathrm{CI}=1.1,2.2$; 
$P=0.01)$ and $0.84(95 \% \mathrm{CI}=0.54,1.3 ; P=0.43)$, respectively. Therefore, ifosfamide in combination with chemotherapy likely provided better effect in patients without metastases than in those with metastases. However, this conclusion should be interpreted with caution because the EICESS-92 study was not powered in each of the subgroups ${ }^{26}$ and the INT-0091 study was not designed for robust comparisons of subgroups. ${ }^{25}$ In this meta-analysis, subgroup analysis in patients with or without metastatic disease was not performed because of limited studies and insufficient data. Thus, betterdesigned, prospective RCTs with complete data should be performed to verify these findings.

The extent of tumor necrosis may have an impact on the EFS outcome. Patients with $>90 \%$ tumor necrosis were classified as good responders (GRs), whereas patients with $<90 \%$ were defined as poor responders (PRs). In the study conducted by Bacci et al, ${ }^{22}$ ifosfamide was administered to patients with poor responses to MTX, cisplatin, and doxorubicin. Higher probability of EFS was observed in patients with GRs than those with PRs (5-year EFS: GR=67\%; PR=56\%). Bacci et $\mathrm{a}^{22}$ indicated that patients with osteosarcoma who exhibited good histologic response likely showed improved EFS when they were subjected to ifosfamide-based chemotherapy. However, this survival benefit was not found in a Phase III trial of Meyers et al, ${ }^{24}$ in which patients were administered with ifosfamide in doses that were similar to those of Bacci et al. ${ }^{22}$ Meyers et al ${ }^{24}$ also found that EFS was not significantly prolonged when ifosfamide was added to a three-drug regimen, which include MTX, cisplatin, and doxorubicin, for the treatment of patients with poor responses. These different results may be attributed to high doses or different schedules used in the two studies.

There are some potential limitations in this meta-analysis. First, our meta-analysis was performed on the basis of six RCTs. The analysis of prognostic factors was based on only three studies. Thus, the conclusion related to the prognostic factors should be interpreted with caution. Second, among the seven included studies, three provided Kaplan-Meier curves instead of HRs with $95 \% \mathrm{CI}$ for survival data. To calculate the estimates, we initially attempted to contact the investigators for the original data; however, this strategy failed. We then extracted the data from Kaplan-Meier curves to compare EFS and OS, which may have resulted in inaccurate data.

In summary, our meta-analysis indicated that ifosfamide combined with chemotherapy significantly improved EFS and OS; furthermore, the combined treatment increased good histologic response rate of patients with osteosarcoma. However, the limited number of studies restricted us from further performing subgroup analysis on the association between prognostic factors and survival outcomes. Thus, large-scale well-designed RCTs are needed to verify these results.

\section{Disclosure}

The authors report no conflicts of interest in this work.

\section{References}

1. Meyers PA, Gorlick R. Osteosarcoma. Pediatr Clin North Am. 1997; 44:973-989.

2. Bielack S, Jurgens H, Jundt G, et al. Osteosarcoma: the COSS experience. Cancer Treat Res. 2009;152:289-308.

3. Antman KH, Ryan L, Elias A, Sherman D, Grier HE. Response to ifosfamide and mesna: 124 previously treated patients with metastatic or unresectable sarcoma. J Clin Oncol. 1989;7:126-131.

4. Jurgens H, Exner U, Kuhl J, et al. High-dose ifosfamide with mesna uroprotection in Ewing's sarcoma. Cancer Chemother Pharmacol. 1989;24 Suppl 1:S40-S44.

5. Magrath I, Sandlund J, Raynor A, Rosenberg S, Arasi V, Miser J. A phase II study of ifosfamide in the treatment of recurrent sarcomas in young people. Cancer Chemother Pharmacol. 1986;18 Suppl 2: S25-S28.

6. Miser JS, Kinsella TJ, Triche TJ, et al. Ifosfamide with mesna uroprotection and etoposide: an effective regimen in the treatment of recurrent sarcomas and other tumors of children and young adults. J Clin Oncol. 1987;5:1191-1198.

7. Kung FH, Pratt CB, Vega RA, et al. Ifosfamide/etoposide combination in the treatment of recurrent malignant solid tumors of childhood. A Pediatric Oncology Group Phase II study. Cancer. 1993;71: 1898-1903.

8. Gentet JC, Brunat-Mentigny M, Demaille MC, et al. Ifosfamide and etoposide in childhood osteosarcoma. A phase II study of the French Society of Paediatric Oncology. Eur J Cancer. 1997;33:232-237.

9. Craft A, Cotterill S, Malcolm A, et al. Ifosfamide-containing chemotherapy in Ewing's sarcoma: the Second United Kingdom Children's Cancer Study Group and the Medical Research Council Ewing's Tumor Study. J Clin Oncol. 1998;16:3628-3633.

10. Paulussen M, Ahrens S, Dunst J, et al. Localized Ewing tumor of bone: final results of the cooperative Ewing's Sarcoma Study CESS 86. J Clin Oncol. 2001;19:1818-1829.

11. Moher D, Liberati A, Tetzlaff J, Altman DG. Preferred reporting items for systematic reviews and meta-analyses: The PRISMA statement. Ann Intern Med. 2009;151:264-269.

12. Jadad AR, Moore RA, Carroll D, et al. Assessing the quality of reports of randomized clinical trials: is blinding necessary? Control Clin Trials. 1996;17:1-12.

13. Kjaergard LL, Villumsen J, Gluud C. Reported methodologic quality and discrepancies between large and small randomized trials in metaanalyses. Ann Intern Med. 2001;135:982-989.

14. Mantel N, Haenszel W. Statistical aspects of the analysis of data from retrospective studies of disease. J Natl Cancer Inst. 1959;22:719-748.

15. DerSimonian R, Laird N. Meta-analysis in clinical trials. Control Clin Trials. 1986;7:177-188

16. Higgins JP, Thompson SG, Deeks JJ, Altman DG. Measuring inconsistency in meta-analyses. BMJ. 2003;327:557-560.

17. Tierney JF, Stewart LA, Ghersi D, Burdett S, Sydes MR. Practical methods for incorporating summary time-to-event data into meta-analysis. Trials. 2007;8:16.

18. Begg CB, Mazumdar M. Operating characteristics of a rank correlation test for publication bias. Biometrics. 1994;50:1088-1101.

19. Egger M, Davey SG, Schneider M, Minder C. Bias in meta-analysis detected by a simple, graphical test. BMJ. 1997;315:629-634. 
20. Le Deley MC, Guinebretiere JM, Gentet JC, et al. SFOPOS94: a randomised trial comparing preoperative high-dose methotrexate plus doxorubicin to high-dose methotrexate plus etoposide and ifosfamide in osteosarcoma patients. Eur J Cancer. 2007;43:752-761.

21. Meyers PA, Schwartz CL, Krailo M, et al. Osteosarcoma: a randomized, prospective trial of the addition of ifosfamide and/or muramyl tripeptide to cisplatin, doxorubicin, and high-dose methotrexate. J Clin Oncol. 2005;23(9):2004-2011.

22. Bacci G, Ferrari S, Tienghi A, et al. A comparison of methods of loco-regional chemotherapy combined with systemic chemotherapy as neo-adjuvant treatment of osteosarcoma of the extremity. Eur J Surg Oncol. 2001;27(1):98-104.

23. Chou AJ, Kleinerman ES, Krailo MD, et al. Addition of muramyl tripeptide to chemotherapy for patients with newly diagnosed metastatic osteosarcoma: a report from the Children's Oncology Group. Cancer. 2009;115:5339-5348
24. Meyers PA, Schwartz CL, Krailo MD, et al. Osteosarcoma: the addition of muramyl tripeptide to chemotherapy improves overall survival - a report from the Children's Oncology Group. J Clin Oncol. 2008;26:633-638.

25. Grier HE, Krailo MD, Tarbell NJ, et al. Addition of ifosfamide and etoposide to standard chemotherapy for Ewing's sarcoma and primitive neuroectodermal tumor of bone. N Engl J Med. 2003;348:694-701.

26. Ferrari S, Ruggieri P, Cefalo G, et al. Neoadjuvant chemotherapy with methotrexate, cisplatin, and doxorubicin with or without ifosfamide in nonmetastatic osteosarcoma of the extremity: an Italian sarcoma group trial ISG/OS-1. J Clin Oncol. 2012;30:2112-2118.

27. Paulussen M, Craft AW, Lewis I, et al. Results of the EICESS-92 Study: two randomized trials of Ewing's sarcoma treatment-cyclophosphamide compared with ifosfamide in standard-risk patients and assessment of benefit of etoposide added to standard treatment in high-risk patients. J Clin Oncol. 2008;26:4385-4393.

\section{Publish your work in this journal}

Drug Design, Development and Therapy is an international, peerreviewed open-access journal that spans the spectrum of drug design and development through to clinical applications. Clinical outcomes, patient safety, and programs for the development and effective, safe, and sustained use of medicines are a feature of the journal, which has also been accepted for indexing on PubMed Central. The manuscript management system is completely online and includes a very quick and fair peer-review system, which is all easy to use. Visit http://www.dovepress.com/testimonials.php to read real quotes from published authors.

Submit your manuscript here: http://www.dovepress.com/drug-design-development-and-therapy-journal 